\title{
Análisis de los procesos de recursos humanos y su influencia en los bonos y prestaciones
}

Analysis of human resource processes and their influence on bonuses and benefits

Analyse des processus des ressources humaines et leur influence sur les bonus et les prestations

Sergio M. Madero Gómez smadero@itesm.mx Técnológico de Monterrey, Monterrey - México

Doctor en Ciencias EconómicoAdministrativas por la Universidad de Deusto en San Sebastián España. Profesor Titular del Departamento de Gestión Empresarial y Talento Humano de la Escuela de Negocios, Ciencias Sociales y Humanidades del Tecnológico de Monterrey, Campus Monterrey, México.

Humberto Peña Rivera hpena@itesm.mx

Técnológico de Monterrey, Monterrey - México

Doctor en Ciencias EconómicoAdministrativas por la Universidad de Deusto en San Sebastián España. Director de la carrera de Lic. en Dirección y Creación de Empresas de la

Escuela de Negocios, Ciencias Sociales y Humanidades del Tecnológico de Monterrey, Campus Monterrey, México.

Artículo de investigación científica y tecnológica Según Clasificación Colciencias

Fecha de recepción: 28/02/2012

Fecha de corrección: 05/06/2012

Fecha de aprobación: $04 / 12 / 2012$

\section{Resumen}

En la actualidad las empresas están siendo cada vez más competitivas, transformando sus procesos con la finalidad de evolucionar, sobre todo en el desarrollo de estrategias para mejorar en su gestión, es decir, en términos de planeación, desarrollo, crecimiento de acuerdo con el entorno en el que interactúa, requiriendo de un enfoque hacia el mejoramiento del talento humano. El presente trabajo tiene como finalidad conocer la relevancia que tienen los diversos procesos de la administración de recursos humanos (políticas salariales, descripciones de puestos, valuaciones de puestos, estructuras de pagos, comunicación entre otras) en el pago de bonos e incentivos a los trabajadores; sin embargo, existe una percepción de la persona hacia el éxito, la motivación o la importancia que representa ese ingreso, para lo cual identificamos que en una muestra de 335 personas, mediante un modelo de ecuaciones estructurales basado en la técnica de mínimos cuadrados parciales [partial least square (PLS)], que efectivamente sí existe una relación entre esas variables, lo que confirma la relevancia que tienen las prácticas de recursos humanos en la administración de las compensaciones, mostrando una correlación con los bonos y prestaciones recibidas por el trabajador $\mathrm{r}(335)=0.72$, p<0.001.

Palabras clave: recursos humanos, compensaciones, bonos, prestaciones, incentivos, motivación.

Código JEL: J2, J3, J5, M1 
Analysis of human resource processes and their influence on bonuses and benefits

\author{
Análisis de los procesos de recursos \\ humanos y su influencia en los bonos y \\ prestaciones
}

Analyse des processus des ressources

humaines et leur influence sur les bonus et les prestations

\section{Abstract}

Today, companies are more competitive, transforming their processes daily to improve, particularly in the development of strategies to impact upon their goals, especially in human talent. This research seeks to know the relevance of the many practices of human resource management (pay policies, job descriptions, job evaluation, pay structure, communication, etc.) in payment of bonuses and incentives to workers; however, the perception exists of the person relating success, motivation or the importance of income through work compensation, for which we identified in a sample of 335 subjects that indeed a relationship does exist among these variables; this was carried out through a model of structural equations, based on the Partial Least Squares, which confirms the relevance of human resource practices in compensation management, revealing a correlation of $r(335)=0.72$, p<0.001 with the bonds and benefits received by the worker.

Keywords: human resource management, compensation, bonuses, payment systems, incentive plans, motivation.

Código JEL: J2, J3, J5, M1
Analyse des processus des ressources humaines et leur influence sur les bonus et les prestations

\author{
Análisis de los procesos de recursos \\ humanos y su influencia en los bonos y \\ prestaciones \\ Analysis of human resource processes and \\ their influence on bonuses and benefits
}

\section{Résumée}

Actuellement, les entreprises sont de plus en plus compétitives, en transformant leurs processus à fin d'évoluer, particulièrement dans le développement de stratégies pour améliorer leur gestion. C'est à dire, en termes de planification, développement, croissance selon l'environnement avec lequel il entre en interaction, ayant besoin d'une approche vers l'amélioration du talent humain. Ce travail a pour objectif de connaître la relevance qui ont les divers processus de la gestion de ressources humaines (politiques de salaire, descriptions de postes, évaluation de postes, structures de paiements, communication parmi d'autres) sur le paiement des bonus et des incitations aux travailleurs, mais il existe une perception de la personne vers le succès, la motivation ou l'importance qui représente ce revenu. C'est pour ça qu'on a identifié, sur un échantillon de 335 personnes à partir d'un modèle d’équations structurelles basée sur la technique des Moindres Carrés Partiels (Partial Least Square -PLS), qu'il existe effectivement une relation entre ces variables, ce qui confirme la relevance des pratiques des ressources humaines dans la gestion des compensations, montrant une corrélation avec les bonus et les prestations reçus par le travailleur. $\mathrm{r}(335)=0.72$, pro.001.

Mots clef: ressources humaines, compensations, bonus, prestations, incitations, motivation.. 


\section{Análisis de los procesos de recursos humanos y su influencia en los bonos y prestaciones}

\section{Introducción}

En la actualidad las empresas en general están siendo cada vez más competitivas y evolucionan en el desarrollo de estrategias para mejorar sus procesos administrativos y operativos en términos de planeación, desarrollo y crecimiento de acuerdo con el entorno que se tiene, requiriendo de una adecuada estrategia que incida en su administración y en el equilibrio de intereses entre todos los involucrados (Azolukwam y Perkins, 2009, Ramlall, 2006).

Además de los problemas operativos, las empresas tienen grandes retos y estándares muy altos por alcanzar para volverse más competitivas; sin embargo, con el apoyo de consultores tanto internos como externos se pueden establecer las bases que las lleven a una adecuada administración, con excelentes sistemas de control y un proceso adecuado para la toma de decisiones, además de implementar diversos esquemas de remuneración económica basada en el desempeño tratando de que tengan un impacto directo tanto en los procesos como en los resultados (Alba, 2012, Madero y Peña, 2008).

En la actualidad existen empresas que no cuentan con una base de referencia para diseñar, desarrollar y poder implementar de forma estratégica esquemas de pagos debidamente profesionalizados, es decir, que exista una plataforma para poder asignar de manera justa los recursos económicos de la empresa y el establecimiento de políticas y procesos desarrollados para el beneficio de los trabajadores y de la empresa misma (Wichitchanya y Durongwatana, 2012, Wattanasupachoke, 2009).

\section{Objetivo de la investigación}

Conocer la relación que existe entre los diversos procesos de recursos humanos que están asociados con la administración de compensaciones, el pago de bonos, incentivos y demás prestaciones.

Identificar y analizar la importancia de los procesos de recursos humanos que inciden en los esquemas de compensaciones.
Identificar si los bonos, incentivos y prestaciones que reciben los trabajadores son percibidos como un aspecto importante en su trabajo, como un motivador o bien como parte del éxito alcanzado en su trabajo.

\section{Marco teórico}

Considerando que el capital humano es uno de los elementos vitales en la transformación y el crecimiento de una empresa (Alba, 2012, Kirkland, 2009), es importante conocer la influencia que tienen las diversas prácticas administrativas y procesos en el desarrollo y crecimiento de una empresa (King-Kauanui, Ngoc y Ashley-Cotleur, 2006).

El entorno donde participan las empresas en este tiempo está muy enfocado con la competitividad, el cumplimiento y logro de resultados, con la eficiencia, con tener una diversidad de herramientas de mejora, con hacer uso correcto de la tecnología y con enfocarse en actividades que sean innovadoras y que generen valor (Gaines, Hoover, Foxx, Matuszek y Morrison,(2012), (Ulrich, 2000), (Galetic y Nacinovic, 2006).

Ante esta situación, la gestión de recursos humanos requiere de una atención especial, es decir, repensar la función que tiene en las empresas y sobre todo enfocarse en el rol a jugar, pues es importante que sea un socio estratégico de las demás áreas funcionales y apoyar a la dirección general al logro de los resultados (Wichitchanya y Durongwatana, 2012), (Lawler, 2006), (Rynes, 2004), (Ordoñez de Pablos, 2004), (Lawler, Levenson y Boudreau, 2004).

La definición, el desarrollo y la implementación de las prácticas y de los modelos de gestión, representan un gran reto en la actualidad, tanto para las pequeñas, como para las grandes empresas, si bien las segundas cuentan con sistemas mucho más estructurados y con mayor apoyo económico, las primeras deben realizar mayores esfuerzos para que sean útiles en sus operaciones diarias y puedan sobrevivir a los embates del mercado, tal como lo mencionan en sus investigaciones Sáinz de Vicuña y Olabarri (2002). 
Las diversas prácticas administrativas que están relacionadas con los procesos de recursos humanos, en particular con la gestión de compensaciones y que se han considerado para el desarrollo de la presente investigación, son las descripciones de puestos, la administración de los pagos, los planes de vida y carrera, la medición del desempeño y la capacitación.

Por eso es importante mencionar el papel que tienen en la gestión de compensaciones los perfiles, las descripciones, los análisis y las valuaciones de puestos, , así como las diversas actividades enfocadas a fortalecer la equidad interna y externa de los pagos (Azolukwan y Perkins, 2009), (Aycan y Kabazacal, 2006), (Horwitz, Teng-Heng y Ahmed, 2003).

Por otra parte, la descripción de puestos y la evaluación del desempeño son básicas en toda organización porque la primera ayuda a delimitar las funciones y sirve de plataforma para la asignación de sueldos y salarios. La evaluación del desempeño es una herramienta para que los trabajadores conozcan cuáles son sus áreas de mejora y en que sus esfuerzos sean reconocidos por la organización (Resurreccion, 2012), (Lacoursière, Fabi y Raymond, 2008), (Okpara y Wynn, 2008), (Hendry, Woodward, Bradley y Perkins, 2000).

Otro aspecto a considerar es la administración sana y eficiente de los salarios. Para ello, la alta dirección debe tener en cuenta que las recompensas se administran y diseñan para que los empleados las perciban de una manera justa y que sean motivantes y puedan mejorar su desempeño (Wickham y O'Donohue, 2012), (Kirkland, 2009). Además diversos autores (Hargis y Bradley, 2011, Zingheim y Schuster, 2002, Clark, 1995/1996, Greene, 1997 y Ledford Jr. y Hawk, 2000] mencionan la importancia de alinear los pagos con las necesidades de la empresa con la finalidad de fortalecer esa función y poder lograr una ventaja competitiva y con ello mejorar la administración de las compensaciones.

Una herramienta importante a considerar en la gestión de recursos humanos es el diseño y desarrollo de los planes de carrera profesional de los empleados que de alguna manera se relacionan con los esquemas de compensaciones, pues tienen impacto en la satisfacción, en el compromiso y en el desempeño de la persona en el puesto (Abdulkadir, Isiaka y Adedoyin ,2012,
McCloskey y Igbaria, 2003, Igbaria y Shayo 1997).

Es relevante mencionar que si las personas en las empresas encuentran posibilidades de promoción en su carrera, mediante un plan de vida y carrera, seguramente se sentirán más satisfechos, manteniendo su intención por permanecer más tiempo en su puesto de trabajo y así contribuir a que los resultados de la organización sean exitosos (Madero, 2010).

Actualmente hay una tendencia en el medio empresarial a la competitividad y productividad, haciendo énfasis en los factores de medición a través de diversos indicadores o métricas (Laureani y Jiju, 2010, Brown y Kelly, 2006). Además las diversas métricas que existen, sirven de base para establecer los sistemas de pagos variables y relacionarlos directamente con el desempeño de las personas (Okes, 2008, Milne, 2007 y Nizar, 2006, Madero, 2006).

De acuerdo con lo que mencionan Lawler, Levenson y Boudreau (2004), Pfeffer y Fong (2005) y Zingheim y Schuster (2007) respecto a que el aumento en la capacidad de la producción del trabajo alcanzado se debe en gran parte al proceso que se tiene para desarrollar mejores capacidades de los trabajadores, eso se adquiere con el entrenamiento, la educación y se agrupan con la experiencia de la persona que hace que el uso de sus capacidades se potencializan.

Ahora bien hay que destacar que los empresarios están conscientes de la necesidad y la importancia de la utilización de diversas técnicas y herramientas para mejorar su gestión e incrementar su competitividad (Peña, 2010, Madero y Peña, 2008, Lansberg, 1983); sin embargo, nos damos cuenta que la mayoría de las empresas solo tienen la intención de utilizarlas, pero quizá se deba a la falta de disponibilidad de recursos para darle seguimiento a su implementación.

En la actualidad muchas organizaciones han decidido utilizar esquemas de pagos alternos para mantener una fuerza laboral de bajo costo, es decir, buscando la implementación de diversas prestaciones para tener ciertos ahorros, así como el diseño de beneficios atractivos para sus empleados, o bien nuevos esquemas de reconocimiento, pues son remuneraciones no monetarias (Mondy, 2010). Por otra parte Carlson, Upton y Seaman (2006), indican la relevancia 
que tienen las prácticas de recursos humanos en el éxito de una empresa, en particular los incentivos y las compensaciones, ya sean de tipo monetario o no.

Muchos de los problemas que existen en las empresas son atribuidos a los aspectos salariales y tienen de alguna manera relación directa con la motivación intrínseca y con la satisfacción de la persona al estar desarrollando su trabajo (Kopelman, Prottas y Davis, 2008, Schwarz, 1989) y tienen sus efectos en el ambiente laboral.

Li-Ping Tang, Shin-Hsiung y Luna-Arocas (2005) mencionan que existen distintas prioridades que las personas tienen por el ingreso que se recibe al momento de estar desempeñando sus actividades y no van sólo en función del objeto económico, sino de otros aspectos que pueden ser profesionales, sociales y familiares que le brindan, según el individuo, un sentido distinto y único de vida.

Chiu, Wai-Mei y Li-Ping Tang (2002), Li-Ping Tang y Chiu (2003) en sus investigaciones utilizan diversos aspectos que los relacionan con el aspecto económico y son la importancia que las personas le otorgan con respecto a otros aspectos que no son solo económicos, también encontramos que el dinero es un factor de motivación, además de que el ingreso que reciben las personas es un sinónimo de éxito, para lo cual consideramos probarlos en la investigación.

\section{Metodología}

El estudio consistió en una investigación con características de tipo cuantitativa, tal como lo indican Hernández, Fernández y Baptista (2010) utilizando como unidad de estudio personas que están trabajando en empresas de la zona metropolitana de Monterrey, Nuevo León, México.

\subsection{Modelo de la investigación}

El modelo de investigación planteado para el desarrollo del presente trabajo, fundamenta que desde el punto de vista de la función de recursos humanos, la implementación de los diversos procesos o prácticas relacionadas con la administración de compensaciones, influyen en el desarrollo y en el establecimiento de los incentivos, bonos y prestaciones que recibe los trabajadores y forman parte de los ingresos por su trabajo, convirtiéndose luego en un factor determinante para las personas.
Figura 1. Modelo particular de la investigación

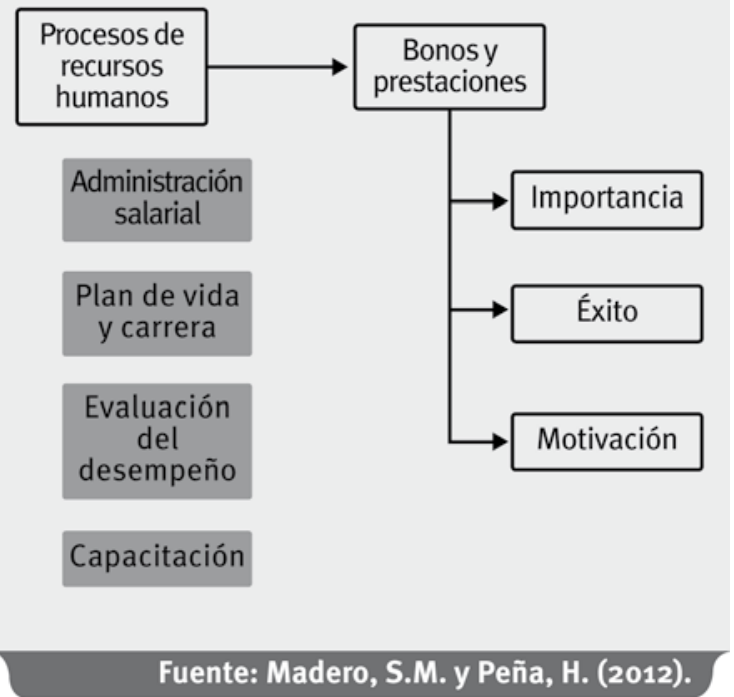

Para lo cual se han definido las siguientes hipótesis:

Hipótesis 1. Los diversos procesos de recursos humanos influyen en la importancia que tienen en las personas los bonos y las prestaciones recibidas en su trabajo.

Hipótesis 2. Los bonos y las prestaciones que se otorgan a los trabajadores influyen para que sean un factor importante en su paquete de compensaciones.

Hipótesis 3. Los bonos y las prestaciones que se otorgan a los trabajadores influyen como un factor de éxito en el desarrollo de su trabajo.

Hipótesis 4. Los bonos y las prestaciones que se otorgan a los trabajadores influyen para ser un factor de motivación en su trabajo.

\subsection{Instrumento de medición}

Para el diseño, elaboración y desarrollo del instrumento de medición a utilizar en la investigación, se ha considerado tomar como base los trabajos realizados por Chiu, Wai-Mei y Li-Ping Tang (2002), Li-Ping Tang y Chiu (2003) y Okpara y Wynn (2008). El cuestionario final fue de 28 preguntas, agrupadas en 4 bloques distribuidos de la siguiente forma:

- Importancia de los procesos de recursos humanos que de alguna manera se relacionan con actividades administrativas de la gestión de compensaciones (compuesto de 6 preguntas, entre las que podemos mencionar: "Comunicar a los empleados el plan 
de compensación de la empresa", "Contar con descripciones de puestos en la empresa", "La evaluación del desempeño del trabajador en la empresa", que fueron evaluadas con una escala Likert del 1 al 5 , siendo 1 No es importante y 5 Sí es muy importante).

- Importancia que tienen los bonos y prestaciones en el sistema de pagos de las personas que trabajan (compuesto de 7 preguntas evaluadas, como por ejemplo "Los bonos que se otorgan en la empresa forman parte importante de la compensación que se recibe", "Los incentivos se pagan de acuerdo con el cumplimiento de metas", "Los bonos y las prestaciones que se otorgan en la empresa ayudan a mejorar el desempeño del empleado", que fueron evaluadas con una escala Likert del 1 al 5, siendo 1 No es importante y 5 Sí es muy importante).

- Percepción de la clasificación del dinero con respecto a los factores "motivación-importancia y éxito (11 preguntas en una escala tipo Likert del 1 al 5, siendo 1 totalmente en desacuerdo y 5 totalmente de acuerdo, como por ejemplo " $\mathrm{El}$ sueldo es un importante factor en mi calidad de vida”, “Mi sueldo refleja mis logros", “Mi sueldo es un motivador para continuar en la empresa").

- Datos sociodemográficos (4 preguntas).

La aplicación se llevó a cabo dentro de las instalaciones de cada una de las empresas donde trabajaban los participantes y se realizó con previa autorización de las autoridades de las organizaciones. Al momento de la aplicación, se les señaló a los participantes que su colaboración sería confidencial y los resultados se utilizarían únicamente para fines académicos y de investigación; las encuestas se aplicaron de manera individual con una duración aproximada de 15 a 20 minutos.

\subsection{Muestra}

Partiendo de la población objeto de estudio previamente establecida y con el propósito de obtener una muestra conforme a los criterios de representatividad, fiabilidad y significancia estadística requeridos para la aplicación del método de ecuaciones estructurales, basado en mínimos cuadrados parciales [partial least squares (PLS)), se procedió a calcular el tamaño de la muestra de acuerdo con el procedimiento sugerido por Barclay, Higgins y Thomson (1995). Según estos autores el tamaño mínimo de la muestra a considerar en un análisis basado en PLS viene dado por la regresión más compleja que se pueda encontrar en el modelo empírico, esto es, primero se debe encontrar cuál de las siguientes dos alternativas es mayor:

a. El número de indicadores en el constructo emergente más complejo, es decir, aquella variable latente que tenga el mayor número de indicadores formativos.

b. El mayor número de constructos antecedentes que preceden a un constructo endógeno.

En el caso particular de este estudio, el constructo emergente más complejo fue el de percepción del dinero, que contiene 11 indicadores.

Para calcular el tamaño mínimo requerido, siguiendo lo sugerido por Barclay, Higgins y Thomson (1995), se procedió a multiplicar el número de indicadores del constructo más complejo (percepción del dinero 11 indicadores) por el factor de 10, dando un tamaño de muestra de 110.

No obstante el tamaño de muestra sugerido desde el tipo de análisis estadístico a utilizar, se obtuvo un tamaño de muestra superior correspondiente a la aplicación de 335 cuestionarios, en la región noreste de México cuyo objeto de estudio son personas que trabajan de las cuales $55 \%$ son hombres y $45 \%$ restante son mujeres, por otra parte $58 \%$ son solteros y $35 \%$ casados, $\mathrm{C} 2(2)=8.1049, \mathrm{p}<.05$.

En lo que respecta a la antigüedad que tienen las personas trabajando en la empresa, $76 \%$ tiene menos de 5 años, mientras que $24 \%$ más de 6 años, c2 (3) $=4.4799$, p〉.01; la antigüedad en el puesto es $80 \%$ tiene menos de 5 años en su puesto de trabajo y $20 \%$ más de 6 años c2 (3) $=8.781$, pr.05.

Es importante mencionar que la investigación se realizó durante el año 2009; entre enero-mayo, se hizo el diseño del instrumento de medición y la recolección de los datos, mientras que en el segundo semestre del año, es decir, junio-diciembre, se realizó el análisis de la información obtenida que se presenta a continuación.

\subsection{Análisis de los datos}

El modelo conceptual propuesto, se probó utilizando el método de modelos de ecuaciones estructurales (MME) basado en la técnica de PLS, pues de acuerdo a lo que mencionan CalvoMora, Leal y Roldán (2006), es una herramienta que ayuda al análisis predictivo de problemas que son complejos además es el que más se 
ajusta a nuestra investigación. También para el análisis de la información, se utilizó el software estadístico SPSS versión 14.0, con la finalidad de identificar las características de la muestra que participó en la investigación, para la validación de cada uno de los constructos y sus componentes se realizó un análisis factorial confirmatorio y un análisis de confiabilidad como soporte en los análisis estadísticos.

\section{Resultados y discusión}

Aún y cuando los parámetros de medida y estructurales se estimaron al mismo tiempo, se analizó e interpretó en dos etapas principales un modelo bajo la técnica de PLS (Berclay, Higgins y Thomson, 1995): 1) La evaluación de la fiabilidad y validez del modelo de medida, y z) la evaluación del modelo estructural, que se evalúa a través de: a) el análisis de la fortaleza del modelo, b) la capacidad predictiva del modelo, y c) la calidad global del modelo.

La Figura 2, muestra el nomograma con los respectivos resultados de los indicadores estadísticos de cada una de las variables latentes y observables que resultaron del análisis estadístico realizado al modelo.

\section{Figura 2. Modelo resultante de la investigación}

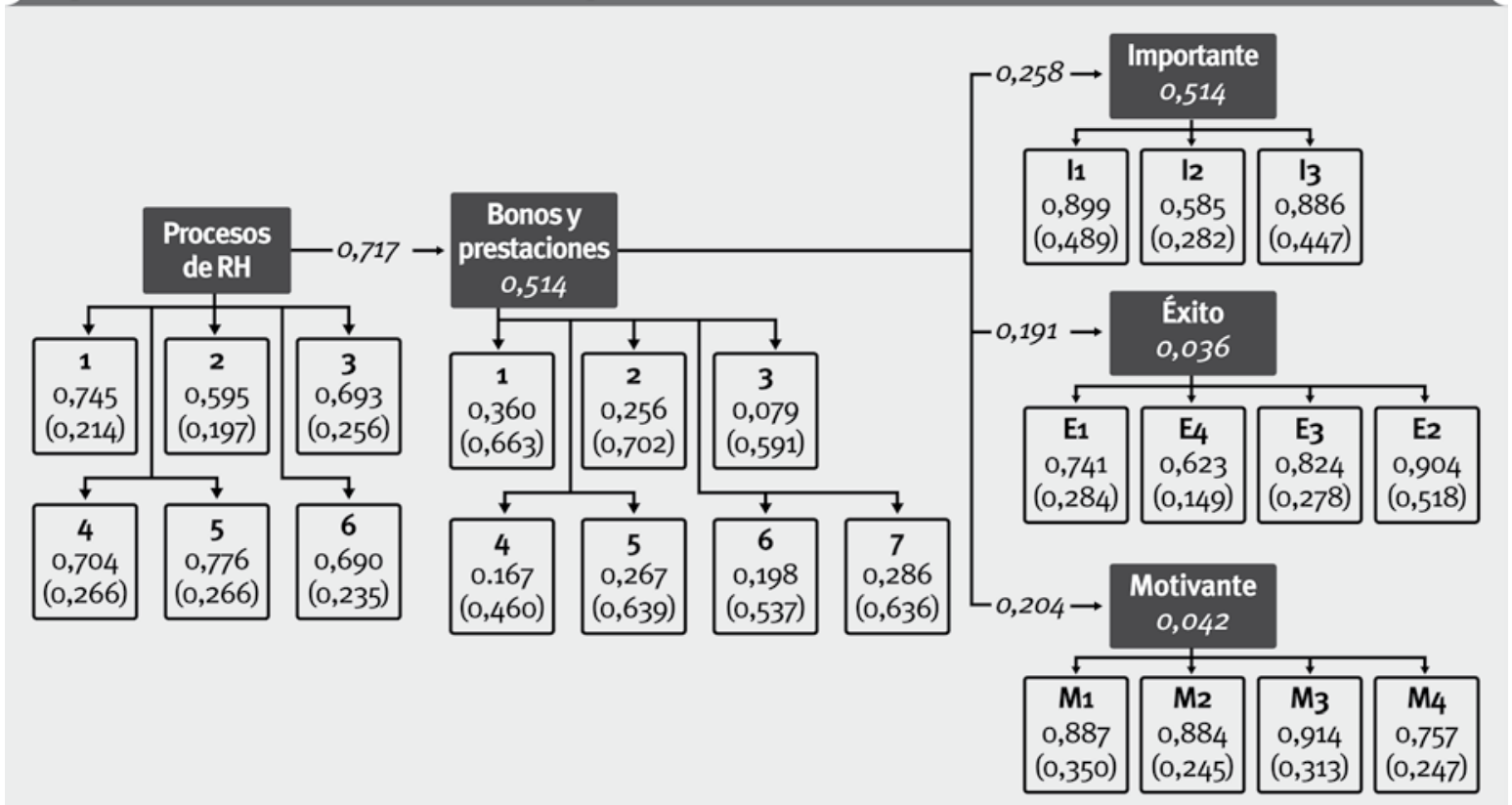

Fuente: Madero, S.M. y Peña, H. (2012).

\subsection{Evaluación del modelo de medida}

El modelo de medida trata de analizar si los conceptos teóricos (constructos) están medidos correctamente a través de las variables observadas (indicadores). El modelo de medida se evalúo a través de tres pruebas:1) fiabilidad individual del ítem, 2) la consistencia interna, y 3) la validez discriminante. Por las características del modelo conceptual, solo se realizó la evaluación para los indicadores reflectivos.

\subsection{Fiabilidad individual del ítem}

Los indicadores reflectivos, explicados antes, son variables manifiestas o valores observados utilizados como medida de un constructo latente que no puede ser medido directamente, son reflejo o manifestación del constructo.
La fiabilidad individual del ítem para indicadores reflectivos se evalúa examinando las cargas (l) o correlaciones simples de los indicadores o medidas con su respectivo constructo. Barclay et al. (1995) afirman que un valor de 0.707 significa que más del $50 \%$ de la varianza de la variable observada es compartida por el constructo.

La Tabla 1 muestra los valores de las cargas de los indicadores reflectivos para los constructos de importancia, éxito y motivación; como se puede observar, todas las cargas de los indicadores presentan valores por encima del nivel recomendado; con excepción del indicador IEMRD y IEMRI, se decidió mantenerlo de acuerdo con la recomendación de los antes autores citados Barclay et al. (1995), quienes establecen que 
cargas mayores de 0.5 pueden mantenerse para dar mayor robustez a la escala.

Así, se puede concluir que los valores indican un adecuado nivel de fiabilidad del modelo de medida, por lo tanto los indicadores miden, efectivamente, la variable latente a la cual han sido ligados.

\subsection{Fiabilidad de constructo}

La valorización de la fiabilidad del constructo se llevó a cabo utilizando el índice de fiabilidad compuesta del constructo desarrollado por Fornell y Larcker (1981). Nunnally (1978) sugiere un valor de 0.70 como un nivel modesto de aceptación en etapas tempranas de investigación, siendo 0.80 un valor más estricto para investigación básica.

En la Tabla 1 aparecen los resultados del cálculo de la medida de la fiabilidad compuesta para cada uno de los constructos y están por encima del nivel de aceptación sugerido, por lo tanto son satisfactorios porque se corrobora que todos los indicadores de un mismo constructo están "unidos".

\begin{tabular}{|c|c|c|c|c|}
\hline $\begin{array}{c}\text { CONSTRUCTO } \\
\text { (Indicador) }\end{array}$ & $\begin{array}{c}\text { Cargas } \\
\text { (A) }\end{array}$ & $\begin{array}{l}\text { Alpha } \\
\text { Cronbach }\end{array}$ & $\begin{array}{l}\text { Fiabilidad } \\
\text { Compuesta }\end{array}$ & $\begin{array}{c}\text { Varianza } \\
\text { Extraida } \\
\text { Media } \\
\text { (AVE) }\end{array}$ \\
\hline IMPORTANCIA & & 0,7249 & 0,8410 & 0,6450 \\
\hline IEMRC (11) & 0,8990 & & & \\
\hline IEMRD (I2) & 0,5851 & & & \\
\hline IEMRE (I3) & 0,8856 & & & \\
\hline ÉXITO & & 0,7909 & 0,8590 & 0,6080 \\
\hline IEMRF(E1) & 0,7407 & & & \\
\hline IEMRG (E2) & 0,9039 & & & \\
\hline IEMRH (E3) & 0,8242 & & & \\
\hline IEMRI (E4) & 0,6234 & & & \\
\hline MOTIVACION & & 0,8833 & 0,9200 & 0,7440 \\
\hline IEMRJ (M1) & 0,8875 & & & \\
\hline IEMRK (M2) & 0,8839 & & & \\
\hline IEMRL $\left(M_{3}\right)$ & 0,9137 & & & \\
\hline IEMRM (M4) & 0,7566 & & & \\
\hline
\end{tabular}

\subsection{Varianza extraída media (AVE)}

En la columna 5 de la Tabla 1 se recogen los resultados correspondientes a la AVE, medida adicional para evaluar la validez del modelo de media. Los valores exceden el nivel de aceptación de .50 sugerido por Fornell y Larcker (1981), lo que significa que más del $50 \%$ de la varianza del constructos se debe a sus indicadores.

\subsection{Validez discriminante}

La validez discriminante se evaluó a través de la matriz de correlaciones entre los constructos (Tabla 2) utilizados en el modelo; se observr que los resultados son validos toda vez que la raíz cuadrada del AVE es superior a los valores de las correlaciones, por lo tanto los constructos son discriminantes entre sí.

\begin{tabular}{|c|c|c|c|c|c|}
\hline & $\begin{array}{l}\text { Bonosy } \\
\text { Presta- } \\
\text { ciones }\end{array}$ & $\begin{array}{l}\text { Impor- } \\
\text { tancia }\end{array}$ & Éxito & $\begin{array}{l}\text { Moti- } \\
\text { vación }\end{array}$ & $\begin{array}{c}\text { Procesos } \\
\text { del RH }\end{array}$ \\
\hline $\begin{array}{l}\text { Bonosy } \\
\text { Prestaciones }\end{array}$ & n.a & & & & \\
\hline Importancia & 0,2580 & 0,8031 & & & \\
\hline Éxito & 0,1910 & 0,5900 & 0,7797 & & \\
\hline Motivación & 0,2040 & 0,4960 & 0,6870 & 0,8626 & \\
\hline $\begin{array}{l}\text { Procesos de } \\
\mathrm{RH}\end{array}$ & 0,7170 & 0,2480 & 0,1100 & 0,1620 & n.a \\
\hline \multicolumn{6}{|c|}{$\begin{array}{l}\text { (a) Los elementos en la diagonal en negrilla, son la raiz } \\
\text { cuadrada del AVE para cada constructo } \\
\text { n.a No aplica }\end{array}$} \\
\hline
\end{tabular}

\subsection{Modelo estructural}

La evaluación del modelo estructural se realizó a través del análisis de la fortaleza de las hipótesis y la calidad global del modelo.

\subsection{Análisis de la fortaleza de las hipótesis}

La Tabla 3 muestra los resultados de los coeficientes beta para cada una de las relaciones (hipótesis). Chin (1998a y 1998b) establece que para que los coeficientes sean interpretados como significativos deben estar por encima del valor de o.3. En el caso especifico de esta investigación, dichos coeficientes son satisfactorios. Así, las hipótesis $\mathrm{H}_{1}, \mathrm{H}_{2}, \mathrm{H}_{3}$ y $\mathrm{H}_{4}$ son soportadas.

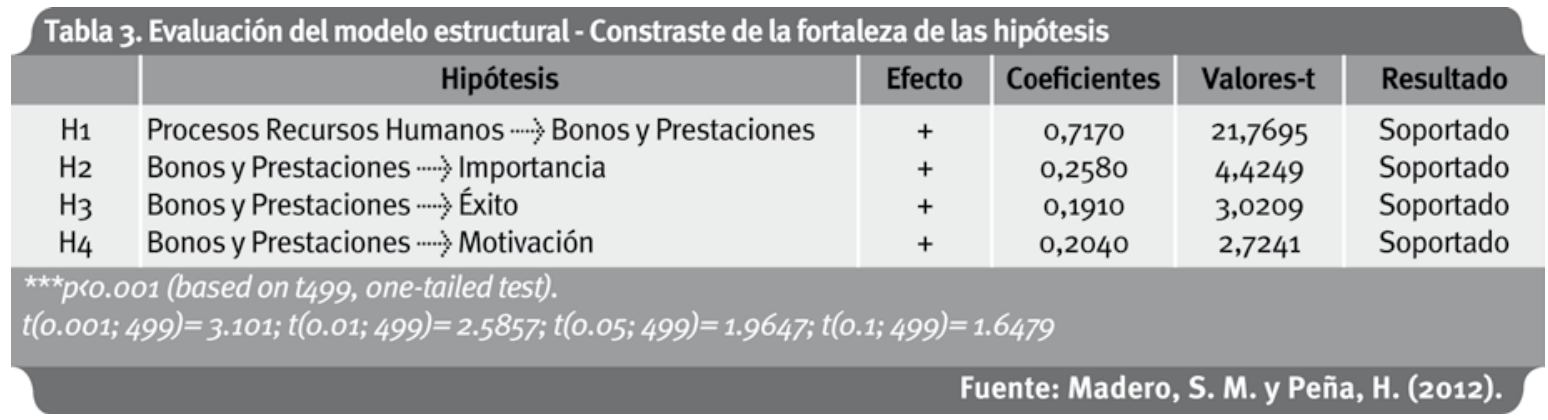




\subsection{Análisis de la calidad global del modelo}

Finalmente, en la Tabla 4 se muestran los resultados correspondientes a la evaluación de la calidad global del modelo. Chin (1998a y 1998b) sugiere utilizar como indicador de medida el Goodness-of-Fit (GOF), este parámetro valora tanto la calidad del modelo de medida de las variables latentes (a través de la media de las AVE de los constructos con indicadores reflectivos) como la calidad del modelo estructural (a través de la media de la $\mathrm{R}^{2}$ de los constructos endógenos). De acuerdo con los resultados, aunque sea bajo, se puede considerar que el modelo en su conjunto es explicado adecuadamente.

Tabla 4. Evaluación del modelo estructural (Análisis de la calidad global del modelo)

Constructo Endógeno

Importancia

Éxito

Motivación

Promedio

(1)

\section{Conclusiones}

A lo largo de la presente investigación se ha expuesto que los procesos de recursos humanos en las organizaciones se enfrentan actualmente a un entorno cada vez más desafiante, en particular los relacionados con los aspectos de la retribución al personal, causado por el incremento en las tendencias de la globalización empresarial, en las que es necesario desarrollar en el personal nuevas competencias laborales para satisfacer las demandas del mercado y las expectativas de los clientes.

Con el desarrollo de la investigación, nos dimos cuenta de la relación y relevancia que tienen los diversos procesos de recursos humanos y que de alguna manera impactan en los sistemas de bonos e incentivos que otorgan las empresas, como son los análisis, las descripciones y las valuaciones de puestos entre otras herramientas más. Bajo este contexto se ha planteado el supuesto de que los procesos de recursos humanos inciden favorablemente en la importancia que las personas le otorgan al pago de algún bono, incentivo o prestación otorgada por la empresa donde trabajan.

De la revisión de la literatura pudimos confirmar la relevancia que tienen los diversos eslabones de unión en los procesos de recursos humanos, como son: 1) la inducción entre la contratación y la capacitación de personal, 2) los planes de vida y carrera entre la capacitación y el desarrollo, 3) las descripciones de puestos entre la capacitación y las compensaciones y finalmente 4) la evaluación del desempeño como unión entre el desarrollo profesional y las compensaciones.

Se pudo comprobar que los resultados alcanzados reflejan la existencia de una relación positiva
Varianza Explicada $\left(\mathbf{R}^{2}\right)$

Goodness-of-fit (GOF)

0,0665

0,0364

0,0417

0,0482

0,1791

Fuente: Madero, S. M. y Peña, H. (2012).

y significativa entre los diversos procesos de recursos humanos que de alguna manera influyen en la importancia que tienen en las personas cuando reciben algún bono o algunas prestaciones en su trabajo. En concreto la aceptación de los bonos y prestaciones está explicada en un $71.7 \%$ por los procesos de recursos humanos analizados.

El supuesto que existe de otorgar bonos y prestaciones a los trabajadores influye de manera positiva en el ingreso total que reciben las personas en su centro de trabajo, se confirma mediante la hipótesis 2 de la investigación, pues efectivamente sí lo consideran como un factor importante en su paquete de compensaciones.

Los sistemas de compensaciones en este caso, formado por el pago de bonos y prestaciones, se convierten en un aliado porque las personas que trabajan así lo llegan a considerar, pues está muy relacionado con el éxito de las personas en el desarrollo de su trabajo, por lo tanto la hipótesis 3 sí se soporta.

Además se ratifica la hipótesis 4 confirmando que los bonos y las prestaciones que se otorgan a los trabajadores influyen como un factor de motivación en las personas. Lo anterior corrobora las afirmaciones de los estudiosos del tema en relación a que los aspectos de compensaciones son un aspecto importante en la motivación de los trabajadores. Por lo tanto, es fundamental que las organizaciones pongan especial atención a estos puntos que se han mencionado para poder mantener al personal en sus centros de trabajos y que las compensaciones no solo sean un factor de atracción sino también de retención. 
En la actualidad los directivos responsables de la administración de las compensaciones deben diseñar estrategias enfocadas para la creación de redes de trabajo con la confianza centrada en el plan de compensaciones que se tiene y que sirva para que se logren los objetivos y metas de la empresa, pues identificamos que los diversos componentes sí son apreciados por las personas.

Además de que la comunicación juega un papel fundamental para que todos los empleados puedan enfocar sus esfuerzos en el mejoramiento de la empresa pues incide favorablemente en el ambiente laboral, en el compromiso y en la motivación.

\section{Posibles proyectos para continuar la investigación}

Es recomendable segmentar las variables relacionadas con las prácticas de recursos humanos y las demás prácticas comunes de la gestión empresarial, profundizando en ellas, tratando de buscar el impacto que tienen directamente en los indicadores y métricas existentes.

Para poder replicar el estudio es conveniente considerar que la crisis económica no sea un factor determinante en los resultados obtenidos y además involucrar variables cuantificables como la retención, permanencia de las personas, niveles de ingreso entre otras más.

Otra variante interesante de investigación en el futuro, podría ser el análisis de la calidad de vida de las personas de acuerdo con la región geográfica donde se lleva a cabo la investigación, al giro que pertenece la empresa, pues el comercio, la industria y los servicios requieren de perfiles de colaboradores distintos, cuyas necesidades tanto individuales como grupales pueden revelar datos muy interesantes, así como el número de personas que ahí trabajan.

Se puede continuar investigando también sobre la influencia que tienen los procesos de recursos humanos y las prácticas de compensaciones en las actitudes de las personas, así como identificar si verdaderamente la remuneración económica es lo más trascendente para las personas que trabajan o bien conocer si existen algunos otros motivadores que se combinan para fortalecer el sentido de pertenencia de un empleado a su organización.

\section{Referencias}

Abdulkadir, D.S., Isiaka, S.B. \& Adedoyin, S.I. (2012). Effects of strategic performance appraisal, career planning and employee participation on_organizational commitment: An empirical study. International Business Research,5 (4) (pp. 124-133).

Alba, S. (2012). La empresa admirada: La receta. México D.F., México: LID Editorial Mexicana.

Aycan, Z. \& Kabasakal, H. (2006). Social contract and perceived justice of workplace practices to cope with financial crisis. Group \& Organization Management, 31 (4) (pp. 469-502).

Azolukwam, V. \& Perkins, S. (2009). Managerial perspectives on HRM in Nigeria: evolving hybridization? Cross Cultural Management, 16 (1) (pp. 62-82).

Barclay, D., Higgins, C. \& Thomson, E. (1995). The Partial Least Squares (PLS) approach to casual modeling: perceval computer adoption and use as an illustration. Technology Studios, Special Issue and Research Methodology, 2 (2) (pp. 285).

Brown, A. \& Kelly, S. (2006). Linking employee research to business performance. Strategic Communication Management, 10 (6) (pp. 2225).

Calvo-Mora, A., Leal, A. \& Roldán, J.L. (2006). Using enablers of the EFQM model to manage institutions of higher education. Quality Assurance in Education, 14 (2) (pp. 99-122).

Carlson, D.S., Upton, N. \& Seaman, S. (2006). The impact of human resource practices and compensation design on performance: an analysis of family-owned SME's. Journal of Small Business Management, 44 (4) (pp. 531-543).

Chin, W.W. (1998a). The partial least approach to structural equation modeling. In: Marcoulides, G.A. (Ed.) Modern methods for business research. Mahwah NJ, USA: Lawrence Erlbaum Associates, Inc.

Chin, W.W. (1998b). Issues and opinion on structural equation modeling, MIS Quarterly, 22 (1) (pp. 7-16).

Chiu, R.K., Wai-Mei, L. \& Li-Ping Tang, T. (2002). Retaining and motivating employees, compen- 
sation preferences in Hong Kong and China. Personnel Review, 31 (4) (pp. 402-431).

Clark, A. (1995). Aligning compensation with business strategy. Employment Relations Today, 22 (4) (pp. 63-71).

Fornell, C. \& Larcker, D.F. (1981). Evaluating structural equation models with unobservable variables and measurement error. Journal of Marketing Research, 18 (1) (pp. 39-50).

Galetic, L. \& Nacinovic, I. (2006). Compensation management in Croatian enterprises: An empirical study. The Business Review, 5 (2) (pp. 204-211).

Greene, R. (1997). Effective variable compensation plans. Analytica Chimica Acta, 6 (1) (pp. 32-39).

Hargis, M.B. \& Bradley, D. (2011). Strategic human resource management in small and growing firms: Aligning valuable resources. Academy of Strategic Management Journal, 10 (2) (pp. 105-125).

Hernández, S,R., Fernández, C. \& Baptista, L. (2010). Metodología de la Investigación ( $5^{\mathrm{a}}$ ed.). México D.F., México: Mc Graw-Hill.

Hendry, C., Woodward, S., Bradley, P. \& Perkins, S. (2000). Performance and rewards: Cleaning out the stables. Human Resource Management Journal, 10 (3) (pp. 46-62).

Horwitz, F., Teng-Heng, C. \& Ahmed, Q. (2003). Finders, keepers? Attracting, motivating and retaining knowledge workes. Human Resource Management Journal, 13 (4) (pp. 23-44).

Igbaria, M. \& Shayo, C. (1997). The impact of race and gender differences on job performance evaluations and career success. Equal Opportunities International, 16 (8) (pp. 12-23).

King-Kauanui, S. Ngoc, S.D. \& Ashley-Cotleur, C. (2006). Impact of human resource management: SME performance in Vietnam. Journal of Developmental Entrepreneurship, 11 (1) (pp. 79-96).

Kirkland, S.D. (2009). Compensation plans that pay back. Business and Economic Review, 55 (2) (pp. 22-23).
Kopelman, R.E, Prottas, D.J. \& Davis, A.L. (2008). Douglas McGregor's theory X and Y: Toward a construct-valid measure. Journal of Managerial Issues, 20 (2) (pp. 255-271).

Lacoursière, R., Fabi, B. \& Raymond, L. (2008). Configuring and contextualising HR systems: An empirical study of manufacturing SMEs. Management Revue, 19 (1/2) (pp. 106-125).

Fabi, B., Raymond, L. \& Lacoursière, R. (2009). Strategic alignment of HRM practices in manufacturing SMEs: a Gestalts perspective. Journal of Small Business and Enterprise Development, 16 (1) (pp. 7-25).

Gaines, C. Hoover, D. Foxx, W. Matuszek, T. \& Morrison, R. (2012). Information systems as a strategic partner in organizational performance. Journal of Management and Marketing Research, 10 (pp. 1-17).

Lansberg, S.I. (1983). Managing human resources in family firms. Organizational Dynamics, 12 (1) (pp. 39-46).

Laureani, A. \& Jiju A. (2010). Reducing employees' turnover in transactional services: a Lean Six Sigma case study. International Journal of Productivity and Performance Management, 59 (7) (pp.688-70o).

Lawler III, E. Levenson, A. \& Boudreau, J. (2004). HR metrics and analytics: Use and impact. Human Resource Planning, 27 (4) (pp. 27-35).

Lawler III, E. (2006). Becoming a key player in business strategy. Workspan, 49 (1) (pp. 10-13).

Ledford, Jr. G., \& Hawk, E. (2000). Compensation strategy: A guide for senior managers. WorldatWork Journal, 9 (1) (pp. 28-38).

Li-Ping T, T. \& Chiu, R.K. (2003). Income, money ethic, pay satisfaction, commitment, and unethical behavior: is the love of the money the root of evil for Hong Kong Employess? Journal of Business Ethics, 46 (1) (pp. 13-30).

Li-Ping Tang, T., Shin-Hsiung T. \& Luna-Arocas, R. (2005). Money profiles: The love of money, attitudes, and needs. Personnel Review, 34 (5) (pp. 603-618).

Madero, S. (2006) Sistemas de retribución variable: Un estudio de la utilización de los bonos 
por desempeño en México. Tesis doctoral. Programa Doctoral en Economía y Dirección de Empresas, Universidad de Deusto. San Sebastián, España.

Madero, S. y Peña, H. (2008). Utilización de herramientas y técnicas de gestión en la PYME: Caso México. Gestión Pública y Empresarial, (12) (pp. 42-64).

Madero, S. (2010). Factores relevantes del desarrollo profesional y de compensaciones en la carrera laboral del trabajador. Contaduría y Administración UNAM, 232 (pp. 109-130).

McCloskey, D. \& Igbaria, M. (2003). Does "out of sight" mean "out of mind"? An empirical investigation of the career advancement prospects of telecommuters. Information Resources Management Journal, 16 (2) (pp. 19-34).

Milne, P. (2007). Motivation, incentives and organizational culture. Journal of Knowledge Management, 11 (6) (pp. 28-38).

Mondy, R. (2010). Administración de recursos humanos (11 ${ }^{\underline{a}}$ ed.). Mexico D.F., México: Pearson Prentice Hall.

Nizar, T. (2006). Subjective performance measures in bonus payouts. Performance Improvement, 45 (8) (pp. 34-41).

Nunally, J. (1978). Psychometric theory ( $2^{\mathrm{a}}$ ed.). New York, USA: McGraw-Hill.

Okes, D. (2008). Driven by Metrics. Quality Progress, 41 (9) (pp. 48-53).

Okpara, J.O. \& Wynn, P. (2008). Human resource management practices in a transition econonomy: challenges and prospects. Management Research News, 31 (1) (pp. 57-76).

Ordoñez de Pablos, P. (2004). Human resource management systems and their role in the development of strate. Journal of European Industrial Training, 28 (pp. 474-489).

Peña, H. (2010). Aprendizaje organizativo y perdurabilidad empresarial: Un estudio de los factores determinantes en las empresas de la región de Monterrey (México). Tesis doctoral. Programa doctoral en Economía y Dirección de Empresas, Universidad de Deusto. San Sebastián, España.
Pfeffer, J. \& Fong, C. T. (2005). Building organization theory from first principles: The self-Eehancement motive and understanding power and influence. Organization Science, 16 (4) (pp. 372-388).

Ramlall, S. (2006). HR competencies and their relationship to organizational practices. Performance Improvement, 45 (5) (pp. 32-44).

Resurreccion, P.F. (2012). Performance management and compensation as drivers of organization competitiveness: The Philippine perspective. International Journal of Business and Social Science,3 (21) (pp. 20-30).

Rynes, S. L. (2004). Where do we go from here?: Imagining new roles for human resources. Journal of Management Inquiry, 13 (3) (pp. 203-213).

Sáinz-de Vicuña, J.M. y Olabarri, J.D. (2002). Utilización de herramientas y técnicas de gestión en la CAPV 2001. Recuperado 8/12/2012 de: https://www.spri.es/wNS/docs/publicaciones/herramo1.pdf

Schwarz R. (1989). Participative decision making. Comparing union-management and management designed incentive pay programs. Group \& Organization Management, 14 (1) (pp. 104-122).

Ulrich, D. (2000). From eBusiness to eHR. HR Human Resource Planning, 23 (2) (pp. 12-21).

Wattanasupachoke, T. (2009). Strategic human resource management and organizational performance: A study of thai enterprises. Journal of Global Business Issues, 3 (2) (pp. 139-148).

Wichitchanya, W. \& Durongwatana, S. (2012). Human resource management and organizational innovation. The Business Review, 20 (1) (pp. 221-227).

Wickham, M. \& O'Donohue, W. (2012). Developing an ethical organization: exploring the role of ethical intelligence, Organization Development Journal, 30 (2) (pp. 9-29).

Zingheim, P. \& Schuster, J. (2002) Aligning total rewards for global economic recovery. Workspan, 45 (5) (pp. 20-26).

Zingheim, P. \& Schuster, J. (2007). Designing pay and rewards in professional services companies. Compensation and Benefits Review, 39 (1) (pp. 55-62). 